\title{
Privacy-Aware Wireless Power Transfer for Aerial Computation Offloading via Colonel Blotto Game
}

\author{
Yao Wang*, Long Zhang*, Minghui Min ${ }^{\dagger}$, Chao Guo ${ }^{\ddagger}$, Vishal Sharma ${ }^{\S}$, and Zhu Han ${ }^{\top} \|$ \\ * School of Information and Electrical Engineering, Hebei University of Engineering, Handan 056038, China \\ $\dagger$ Department of Communication Engineering, Xiamen University, Xiamen 361005, China \\ $\ddagger$ Department of Communication Engineering, Beijing Electronics Science and Technology Institute, Beijing 100070, China \\ $\S$ ISTD Pillar, Singapore University of Technology and Design (SUTD), Singapore 487372, Singapore \\ IDepartment of Electrical and Computer Engineering, University of Houston, Houston, TX 77004, USA

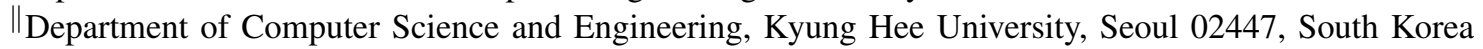

\begin{abstract}
In this paper, a laser-powered aerial multi-access edge computing (MEC) architecture is proposed, where a highaltitude platform (HAP) integrated with an MEC server transfers laser energy to charge aerial user equipments (AUEs) for offloading their computation tasks to the HAP. Particularly, we identify a new privacy vulnerability caused by the transmission of wireless power transfer (WPT) signaling in the presence of a malicious smart attacker (SA). To address this vulnerability, the interaction between the HAP and SA in their allocation of tile grids as charging points to the AUEs in laser-enabled WPT is formulated as a Colonel Blotto game (CBG), which models the competition of two players for limited resources over multiple battlefields for a finite time horizon. Moreover, the utility function that each player receives over a battlefield is developed by revealing the tradeoff between privacy protection level and energy consumption of each AUE. We further obtain the mixed-strategy Nash equilibrium for the modified CBG with asymmetric players. Simulation results are presented to show the effectiveness of this game framework.
\end{abstract}

\section{INTRODUCTION}

With the rapid proliferation of smart user equipments (UEs) on the ground, a multitude of mobile applications are emerging and gaining popularity, such as extended reality, autonomous driving, connected machines, etc. In addition to the terrestrial UEs, unmanned aerial vehicles (UAVs) acting as the aerial UEs (AUEs) have also drawn increasing interests recently for both civilian and commercial applications, e.g., aerial surveillance, disaster response, flying cars, and so on [1]-[3]. Among them, most of the applications are not only computation-intensive but also latency-sensitive. For instance, a huge amount of data collected by the AUEs in disaster sensing missions, e.g., reconnaissance, situational awareness, and mapping, generally consume extensive computational resources.

However, the constrained computing capability of the AUEs is usually difficult to fulfill the computation requirements of these applications. To tackle this issue, multi-access edge computing (MEC) is envisioned as a promising approach to provide the cloud-computing capability in close proximity to the users [4]. By enabling the users to offload their computation tasks to the network edges for execution, MEC has potentials

Corresponding author: Long Zhang (email: zhanglong@ hebeu.edu.cn).

This work was supported in part by NSFC (61901115), Hebei Program for Top-notch Young Talents (BJ2017037), and NSF of Hebei (F2019402206). to bring many advantages including reduced latency, enhanced security, improved energy saving, enhanced user experience, etc. To provide the edge computing resources for the AUEs, one general solution is to integrate the MEC servers at the edge of terrestrial networks, e.g., gNBs in 5G wireless systems. However, it is impractical and uneconomical to deploy such a kind of terrestrial infrastructure integrated with MEC servers for the AUEs when they are distributed above the challenging hard-to-reach environments, e.g., disaster scenarios and ocean areas. An alternative is to extend the computation resources to airspace for providing efficient and flexible computing services for the AUEs. Compared to the UAVs in lower airspace, highaltitude platforms (HAPs) in the stratosphere are more suitable to offer elastic computing services due to larger area coverage, bigger payload capacity, and longer endurance [3]. To this end, the integration of the HAPs with MEC servers motivates the prospect of implementing the aerial computation offloading for the HAP-connected AUEs.

Although HAP-aided MEC can reduce the energy consumption effectively, computation offloading may be still interrupted due to AUE's limited energy storage. An attractive solution is to apply far-field wireless power transfer (WPT) to provide a controllable, sustainable, and longer-range energy supply for the AUEs [5]. Recently, the integration of far-field WPT into the MEC system has gained many research interests, aiming to improve the system performance by optimizing task offloading and resource allocation jointly [6]-[8]. However, these works mainly focus on the use of radio frequency (RF)based WPT at the terrestrial or aerial infrastructure to prolong the lifetime of energy-limited UEs on the ground.

Unlike the RF-based WPT, the laser-enabled WPT is able to deliver much more energy to the users with high energy concentration over long distances [9]. It has been demonstrated by the JAXA that an orbiting satellite with a solar condenser can quickly distribute tens of $\mathrm{kW}$ class laser power on demand to the target areas over the Earth's surface [10]. Therefore, we propose to employ the laser-enabled WPT for HAP-aided MEC to develop a novel paradigm of the laser-powered aerial MEC system, wherein a laser transmitter is mounted at the HAP. With this setup, the HAP transmits laser energy to charge the AUEs from the sky, and the AUEs utilize the harvested 
energy to support the flights and complete the computation tasks. Several recent works are devoted to the application of laser-enabled WPT as the energy supply for the UAVs [5], [9], [11] and the Internet of Things (IoT) devices [12]. However, the above studies exploit the laser-enabled WPT only to the scenario of UAV communications and IoT, and do not capture the effect of laser-based WPT on the HAP-aided MEC.

Meanwhile, to obtain an effective aerial location for charging, each AUE needs to transmit a WPT signaling to the HAP on a control channel via air-to-air (A2A) uplink transmission. However, the WPT signaling delivery in open radio environment is also exposed to smart attacks [13], as a malicious smart attacker (SA) with a smart radio device flexibly chooses the attack mode and strength by monitoring the WPT activity. Moreover, the privacy of AUEs' charging locations can also be compromised by observing the WPT signaling dissemination at the SA. Hence, enhanced security and privacy mechanisms are important for the WPT since a minor compromise may result in a major security problem, e.g., the leakage of charging location information for AUEs. This, however, may allow the SA stealthily infer and track the AUEs' locations and launch attacks for the WPT. To identify a balance between privacy and performance in HAP-aided MEC, a privacy-aware laserenabled WPT problem is formulated in this work based on the framework of Colonel Blotto game (CBG). To the best of our knowledge, this is the first work that considers the aerial location privacy for laser-enabled WPT in HAP-assisted aerial MEC system. Main contributions of our work include:

- We develop a privacy-aware laser-powered aerial MEC framework, which achieves the enhanced location privacy for AUEs under a multi-tier grid-based spatial structure.

- We formulate the interaction between the HAP and the SA in tile grid allocation for the potential charging points to AUEs as a CBG, which models the competition of two players for limited resources over a set of battlefields.

- We design the utility function obtained by each player on a battlefield by capturing the balance between privacy protection level and energy consumption in the aerial MEC system. Moreover, the solution of the CBG corresponds to the mixed-strategy Nash equilibrium point, which is derived for asymmetric players.

The remainder of the paper is organized as follows. Section II describes the system model. In Section III, we formulate the Colonel Blotto game between HAP and SA, and obtain a solution of the game. Simulation results are presented in Section IV, followed by concluding remarks in Section V.

\section{SySTEM MODEL}

\section{A. System Overview}

We consider a laser-powered aerial MEC system as shown in Fig. 1, utilize the aerodynamic lift to maintain circle above the coverage area where a multi-antenna quasi-stationary HAP is located at an altitude of $L_{\mathrm{H}}$ above the Earth's surface in the stratosphere to provide coverage for $N$ single-antenna flying AUEs in a given airspace $\Omega$ within a finite time horizon $T$. Particularly, a laser transmitter and an MEC server are

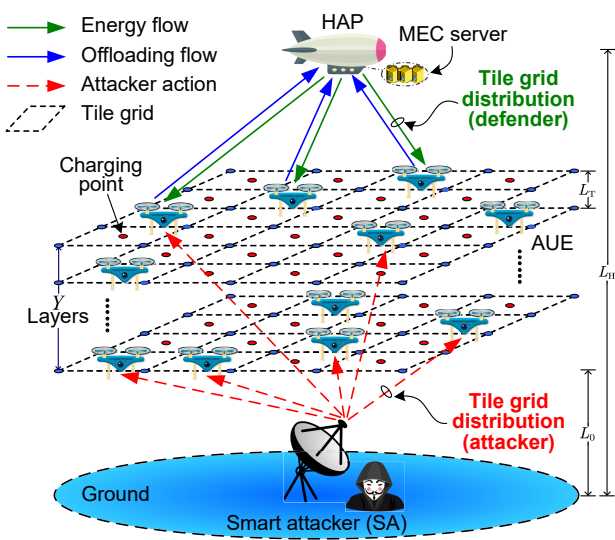

Fig. 1. The considered aerial MEC system in the presence of an SA.

integrated at the HAP. Each AUE has a computation-intensive task to be completed. With the harvest-then-transmit mode, the HAP delivers laser energy to charge the AUEs, and each AUE then uses the harvested energy to maintain its flight and accomplish its computation task via executing locally or offloading to the HAP. We denote the set of AUEs as $\mathcal{N}=\{1,2, \cdots, N\}$. To achieve the laser-enabled WPT, each AUE must send a WPT signaling to the HAP via a control channel in the presence of a malicious SA with a fixed ground location. By using a smart radio device, the SA may passively overhear the WPT signaling to launch attacks for WPT.

Without losing generality, the time horizon $T$ is equally divided into $K$ time slots with length $\delta=\frac{T}{K}$. Then the distance between the HAP and AUE $n$ at slot $k$ is given by $d_{n}[k]$, for $n \in \mathcal{N}$. For simplicity, the considered airspace $\Omega$ is discretized into a multi-tier spatial structure consisting of regular square tile grids, as depicted in Fig. 1. Let us suppose that there are $Y$ tiers for the discretized structure and each tier is composed of $Q \times Q$ square tile grids, for $Q \times Q \times Y \gg N$. Besides, all the tiers are assumed to be all evenly spaced by vertical distance, denoted by $L_{\mathrm{T}}$, for $(Y-1) L_{\mathrm{T}} \ll L_{\mathrm{H}}$. We define $L_{0}$ as the vertical distance between the lowest tier and Earth's surface. Each tile grid in every tier is adopted as a spatial location to charge an AUE via the laser-enabled WPT at a given slot. Note that the location of charging point for AUE is at the center of tile grid. When receiving the WPT signaling from an AUE, the HAP sends the tier-related location data of charging point of associated tile grid to that AUE at a given slot. Meanwhile, the SA performs the attacks by sending the false location data of charging point to that AUE, leading to the unsuccessful WPT.

\section{B. Laser-Enabled WPT Model}

With the harvest-then-transmit mode, the HAP wirelessly transfers laser energy concurrently to $N$ AUEs at each slot by pointing $N$ laser beams to $N$ AUEs, respectively. We assume that the LOS A2A laser link is established between the HAP and each AUE, and the HAP adopts a fixed transmit power $\beta$ of the associated laser beam during time horizon $T$. Thus, the actual energy harvested by AUE $n$ at slot $k$ is given by [9]

$$
E_{n}^{\mathrm{H}}[k]=\tau \delta \beta \frac{A}{\left(D+d_{n}[k] \Delta \theta\right)^{2}} \chi e^{-d_{n}[k] \zeta},
$$


where $\tau \in(0,1)$ is the laser energy conversion efficiency, $A$ is the area of the receiver telescope or collection mirror, $D$ is the size of the initial laser beam, $\Delta \theta$ is the angular spread, $\chi$ is the combined transmission receiver optical efficiency, and $\zeta$ is the attenuation coefficient of the medium. During time horizon $T$, the actual energy harvested by AUE $n$ can be obtained as

$$
E_{n}^{\mathrm{H}}=\sum_{k=1}^{K} E_{n}^{\mathrm{H}}[k]=\sum_{k=1}^{K} \frac{\tau \delta \beta A \chi e^{-d_{n}[k] \zeta}}{\left(D+d_{n}[k] \Delta \theta\right)^{2}} .
$$

\section{Communication Model}

To offload its computation task to the HAP through the A2A uplink transmission, we assume that each AUE is assigned to an orthogonal subchannel over the resource block (RB). Each subchannel has an equally-sized bandwidth of $W$. Note that the co-channel interference during the uplink transmission is completely avoided. We also suppose that the HAP operates in the Ka-band sharing the same RB based on the ITU-R spectrum regulation [3]. For simplicity, the A2A transmission links between AUEs and HAP are dominated by the LOS links, wherein the channel fading depends on both free space path loss and miscellaneous atmospheric loss. With this in mind, the path loss between AUE $n$ and the HAP at slot $k$ can be calculated by

$$
l_{n}[k]=\left(\frac{4 \pi \phi_{n} d_{n}[k]}{c}\right)^{\alpha}+l_{\mathrm{A}},
$$

where $\phi_{n}$ is the carrier frequency of subchannel for AUE $n$, $c$ is the speed of light, $\alpha \geq 2$ is the path loss exponent, and $l_{\mathrm{A}}$ is the atmospheric loss depending on the effect of oxygen and water vapour on $\mathrm{A} 2 \mathrm{~A}$ transmission link. Thus, the channel gain between AUE $n$ and the HAP at slot $k$ can be written as $g_{n}[k]=\frac{1}{l_{n}[k]}$. As a result, the achievable uplink rate of AUE $n$ to the HAP at slot $k$ can be given as

$$
R_{n}^{\mathrm{U}}[k]=W \log _{2}\left(1+\frac{p_{n}[k] g_{n}[k]}{\sigma^{2}}\right),
$$

where $p_{n}[k]$ is the transmit power of AUE $n$ to the HAP at slot $k$, and $\sigma^{2}$ is the variance of the AWGN at the HAP.

\section{Computing Model}

In this paper, we assume that the AUEs adopt a partial computation offloading rule. That is, the computation task of each AUE can either be executed locally by itself, or be offloaded via the A2A uplink transmission to and executed by the HAP. We use the number of input-bits including the program codes and input parameters to describe the computation task for each AUE. Without loss of generality, the task of AUE $n$ at slot $k$ is defined by a two-tuple $\left\{B_{n}[k], \xi_{n}[k]\right\}$, where $B_{n}[k]$ is the number of total input-bits for AUE $n$, and $\xi_{n}[k]$ is the number of input-bits for offloading from AUE $n$ to the HAP.

1) Local Execution: Given each time slot, we denote by $\varpi_{n}$ the number of CPU cycles required to process one bit of input data at $\operatorname{AUE} n$, and let $f_{n}$ be the local computing capability (in CPU cycles/s) of AUE $n$. Therefore, the computing rate (in bps) for local execution at AUE $n$ at slot $k$ is determined by $r_{n}^{\mathrm{L}}[k]=\frac{f_{n}}{\varpi_{n}}$, which is also equivalent to the local computing rate of $\mathrm{AUE} n$ during time horizon $T$, i.e.,

$$
r_{n}^{\mathrm{L}}=r_{n}^{\mathrm{L}}[k]=\frac{f_{n}}{\varpi_{n}} .
$$

Based on the CPU architecture, the power consumption of AUE $n$ for local computing is given by $q_{n}^{\mathrm{L}}=\kappa f_{n}^{3}$, where $\kappa$ is an effective switched capacitance depending on chip architecture of CPU for each AUE [7]. As such, the energy consumption of AUE $n$ for local computing at slot $k$ is given as $E_{n}^{\mathrm{L}}[k]=\kappa f_{n}^{3} \delta$. Accordingly, during time horizon $T$, the energy consumption of AUE $n$ for local computing is specified by

$$
E_{n}^{\mathrm{L}}=\sum_{k=1}^{K} E_{n}^{\mathrm{L}}[k]=\sum_{k=1}^{K} \kappa f_{n}^{3} \delta .
$$

2) Computation Offloading: By considering the A2A uplink transmission overhead (e.g., encryption and packet header), denoted by $\eta_{n}$, the actual number of input-bits of AUE $n$ to be offloaded at slot $k$ is given as $\eta_{n} \xi_{n}[k]$. Then the uplink transmission latency of AUE $n$ for offloading the task to the HAP at slot $k$ can be expressed as $t_{n}^{\mathrm{U}}=\frac{\eta_{n} \xi_{n}[k]}{R^{\mathrm{U}}[k]}$. So the energy consumption for the uplink transmission of AUE $n$ offloading the task at slot $k$ is written by $E_{n}^{\mathrm{U}}[k]=p_{n}[k] t_{n}^{\mathrm{U}}$. As a result, the energy consumption in the uplink transmission for AUE $n$ during time horizon $T$ is modeled as

$$
E_{n}^{\mathrm{U}}=\sum_{k=1}^{K} E_{n}^{\mathrm{U}}[k]=\sum_{k=1}^{K} \frac{\eta_{n} \xi_{n}[k] p_{n}[k]}{R_{n}^{\mathrm{U}}[k]} .
$$

\section{E. Flight Energy Consumption Model}

The flight energy consumption for AUEs mainly depends on their propulsion energy consumption to maintain airborne and support their velocities and accelerations. We assume that each AUE has the same flight energy consumption for the sake of simplicity. As in [14], the flight energy consumed by AUE $n$ during time horizon $T$ can be determined by

$$
\begin{aligned}
E_{n}^{\mathrm{F}}= & \frac{1}{2} m\left(\|\mathbf{v}[K]\|^{2}-\|\mathbf{v}[1]\|^{2}\right)+\sum_{k=1}^{K} \delta\left(\ell_{1}\|\mathbf{v}[k]\|^{3}\right) \\
& +\sum_{k=1}^{K} \delta\left(\frac{\ell_{2}}{\|\mathbf{v}[k]\|}\left(1+\frac{\|\mathbf{a}[k]\|^{2}-\frac{\left(\mathbf{a}^{\mathrm{T}}[k] \mathbf{v}[k]\right)^{2}}{\|\mathbf{v}[k]\|^{2}}}{g^{2}}\right)\right),
\end{aligned}
$$

where $\ell_{1}$ and $\ell_{2}$ are parameters related to the weight, wing area, air density of AUE, $m$ is the mass of AUE consisting of all the payloads, $g$ is the acceleration of gravity, the superscript $\mathbf{a}^{\mathrm{T}}$ is the transpose of vector $\mathbf{a}$, and $\mathbf{v}[k]$ and $\mathbf{a}[k]$ are the AUE's velocity and acceleration vectors at slot $k$, respectively.

\section{GAME FORMULATION AND SOLUTION}

In this section, we employ the $\mathrm{CBG}$ framework to formulate the interaction between the HAP (defender) and the SA in their competitive allocation of tile grids over multiple AUEs, and describe the solution to the proposed tile grid allocation game.

\section{A. Colonel Blotto Game Formulation}

Definition 1 (Pure Strategy): A Colonel Blotto game with pure strategy for tile grid allocation of $N$ AUEs during time horizon $T$ is formulated as a 4-tuple:

$$
\mathfrak{C} \mathfrak{B}_{1}=\left\{\mathcal{P}, \mathcal{N},\left\{\mathscr{X}_{n}^{[T]}\right\}_{n \in \mathcal{N}},\left\{u_{\mathscr{X}, n}^{[T]}\right\}_{n \in \mathcal{N}}\right\}
$$


- Player set $\mathcal{P}: \mathcal{P}=\{H, S\}$, wherein there are two players, $H$ (HAP) and $S$ (SA), that compete by simultaneously distributing the tile grids over $N$ AUEs.

- Battlefield set $\mathcal{N}$ : A battlefield stands for an AUE. There are $N$ independent battlefields, denoted by set $\mathcal{N}$.

- Action space $\left\{\mathscr{X}_{n}^{[T]}\right\}_{n \in \mathcal{N}}$ : Each player allocates the number of tile grids $\mathscr{X}_{n}^{[T]}$ to AUE $n$ during $T$, for $\mathscr{X} \triangleq$ $H$ (HAP) and $\mathscr{X} \triangleq S$ (SA). The distribution vectors of HAP and SA are represented by $\mathbf{H}[T]=\left\{H_{n}^{[T]}\right\}_{n \in \mathcal{N}}$ and $\mathbf{S}[T]=\left\{S_{n}^{[T]}\right\}_{n \in \mathcal{N}}$, respectively. The total number of tile grids allocated by HAP and SA over battlefield set $\mathcal{N}$ during $T$ are given as $Z_{H}^{[T]}$ and $Z_{S}^{[T]}$, respectively.

- Utility function $\left\{u_{\mathscr{X}, n}^{[T]}\right\}_{n \in \mathcal{N}}$ : The utility obtained by each player from battlefield $n$ during $T$ is characterized as $u_{\mathscr{X}, n}^{[T]}$. The total utilities of the HAP and the SA are expressed by $u_{H}^{[T]}=\sum_{n=1}^{N} u_{H, n}^{[T]}$ and $u_{S}^{[T]}=\sum_{n=1}^{N} u_{S, n}^{[T]}$, respectively. Considering that the $\mathrm{CBG}$ is a type of zerosum game, the utilities of two players always add up to zero, i.e., $u_{H, n}^{[T]}=-u_{S, n}^{[T]}$ and $u_{H}^{[T]}=-u_{S}^{[T]}$.

For $\mathfrak{C B}_{1}$, each battlefield is won by the player that allocates the larger number of tile grids to that battlefield [15]. To this end, the idea of the proposed privacy-aware laser-enabled WPT in this work is to compare the number of assigned tile grids for each battlefield between two players during $T$. Specifically, when $H_{n}^{[T]}>S_{n}^{[T]}$, the HAP wins battlefield $n$. In this way, the privacy of charging location for AUE $n$ is protected, such that AUE $n$ can normally harvest the laser energy and conduct the computation offloading. Otherwise, when $H_{n}^{[T]} \leq S_{n}^{[T]}$, the SA wins battlefield $n$. The SA then launches attack for the WPT from the HAP to AUE $n$. On the whole, the player who wins more battlefields than its opponent will win the game. Hence, our goal is to maximize the number of times that the HAP wins over $N$ battlefields in $\mathfrak{C B}_{1}$, which enhances the privacy level of WPT and improves the total utilities of the HAP.

To obtain a balance between privacy protection level and energy consumption of the AUE in the laser-powered aerial MEC system, we define the utility that the HAP receives on battlefield $n$ during time horizon $T$ as follows

$$
\begin{aligned}
& u_{H, n}^{[T]}(\mathbf{H}, \mathbf{S})=-u_{S, n}^{[T]}(\mathbf{H}, \mathbf{S}) \\
= & \begin{cases}\lambda_{1} \frac{r_{n}^{\mathrm{L}}\left(E_{n}^{\mathrm{H}}-E_{n}^{\mathrm{F}}-E_{n}^{\mathrm{U}}\right)}{q_{n}^{\mathrm{L}} \sum_{n=1}^{N} B_{n}[k]}-\lambda_{2}\left(E_{n}^{\mathrm{L}}+E_{n}^{\mathrm{U}}\right), & H_{n}^{[T]}>S_{n}^{[T]}, \\
0, & H_{n}^{[T]} \leq S_{n}^{[T]},\end{cases}
\end{aligned}
$$

where $\lambda_{1}$ and $\lambda_{2}$ are the positive weighting factors.

To simplify the presentation, let us use $\epsilon_{n}^{1}$ to stand for the utility that the HAP receives on battlefield $n$ when $H_{n}^{[T]}>S_{n}^{[T]}$. We further define $\epsilon_{n}^{2}$ as the utility that the HAP receives on battlefield $n$ when $H_{n}^{[T]} \leq S_{n}^{[T]}$. For clarity of exposition, we then introduce a binary variable to represent the association relationship between $H_{n}^{[T]}$ and $S_{n}^{[T]}$ during $T$, i.e.,

$$
F\left(H_{n}^{[T]}, S_{n}^{[T]}\right)= \begin{cases}1, & H_{n}^{[T]}>S_{n}^{[T]} \\ 0, & H_{n}^{[T]} \leq S_{n}^{[T]} .\end{cases}
$$

The total utilities obtained by the HAP over battlefield set $\mathcal{N}$ during $T$ in $\mathfrak{C B}_{1}$ with pure strategy is thus written as

$$
\begin{aligned}
& u_{H}^{[T]}(\mathbf{H}, \mathbf{S})=-u_{S}^{[T]}(\mathbf{H}, \mathbf{S}) \\
= & \sum_{n=1}^{N}\left\{\epsilon_{n}^{1} F\left(H_{n}^{[T]}, S_{n}^{[T]}\right)+\epsilon_{n}^{2}\left[1-F\left(H_{n}^{[T]}, S_{n}^{[T]}\right)\right]\right\} .
\end{aligned}
$$

However, it has been revealed that the pure-strategy Nash equilibrium (NE) for $\mathfrak{C B}_{1}$ does not always exist [15]. Indeed, given any pure strategies, the SA can always modify its tile grid allocation strategy accordingly to improve its utility and win the game. Alternatively, for solving the CBG-based tile grid allocation game, a more general method is to find the mixed-strategy NE solution. In the case of mixed strategies, each player selects a probability distribution over the action space to maximize its potential expected total utilities.

Definition 2 (Mixed Strategy): A Colonel Blotto game with mixed strategy for tile grid allocation of $N$ AUEs during time horizon $T$ is formulated as a 4-tuple:

$$
\mathfrak{C B}_{2}=\left\{\mathcal{P}, \mathcal{N},\left\{\mathscr{X}_{n, r}^{[T]}\right\}_{n=1, r=0}^{N, Z_{\mathscr{X}}^{[T]}},\left\{U_{\mathscr{X}}^{[T]}(\mathbf{h}, \mathbf{s})\right\}\right\},
$$

- Action space $\left\{\mathscr{X}_{n, r}^{[T]}\right\}_{n=1, r=0}^{N, Z_{\mathscr{X}}^{[T]}}$ : The probability that a player allocates $r$ tile grids to AUE $n$ during $T$ is defined by $\mathscr{X}_{n, r}^{[T]}=\operatorname{Pr}\left(\mathscr{X}_{n}^{[T]}=r\right)$, for $\mathscr{X} \triangleq H$ (HAP) and $\mathscr{X} \triangleq S$ (SA). The probability distribution vectors of the HAP and the SA are denoted as $\mathbf{h}[T]=\left\{H_{n, r}^{[T]}\right\}_{n=1, r=0}^{N, Z_{H}^{[T]}}$ and $\mathbf{s}[T]=\left\{S_{n, r}^{[T]}\right\}_{n=1, r=0}^{N, Z_{S}^{[T]}}$, respectively.

- Utility function $\left\{U_{\mathscr{X}}^{[T]}(\mathbf{h}, \mathbf{s})\right\}$ : The expected total utilities obtained by each player over battlefield set $\mathcal{N}$ during $T$ is described as $U_{\mathscr{X}}^{[T]}(\mathbf{h}, \mathbf{s})$. Due to the fact that the CBG is a type of zero-sum game, the expected total utilities of two players always add up to zero, i.e.,

$$
\begin{aligned}
& U_{H}^{[T]}(\mathbf{h}, \mathbf{s})=-U_{S}^{[T]}(\mathbf{h}, \mathbf{s}) \\
= & \underset{\mathbf{S} \sim \mathbf{S}}{\mathbf{h} \sim \mathbf{H}}\left[\sum_{i=1}^{N}\left\{\epsilon_{n}^{1} F\left(H_{n}^{[T]}, S_{n}^{[T]}\right)+\epsilon_{n}^{2}\left[1-F\left(H_{n}^{[T]}, S_{n}^{[T]}\right)\right]\right\}\right] .
\end{aligned}
$$

Based on Definition 2, we define $\psi_{i, r}^{[T]}$ and $\varphi_{i, r}^{[T]}$ by the $i$-th highest probability of $H_{n, r}^{[T]}$ and the $i$-th highest probability of $S_{n, r}^{[T]}$, respectively, for $i \in \mathcal{N}$. Then the mixed-strategy action set for the HAP and the SA can be determined by

$$
\begin{gathered}
\boldsymbol{\Pi}_{H}=\left\{\left\{\psi_{i, r}^{[T]}\right\}_{i=1, r=0}^{N, Z_{H}^{[T]}} \mid \psi_{i, r}^{[T]} \geq H_{n, r}^{[T]}, \sum_{r=0}^{Z_{H}^{[T]}} \psi_{i, r}^{[T]}=1\right\}, \\
\boldsymbol{\Pi}_{S}=\left\{\left\{\varphi_{i, r}^{[T]}\right\}_{i=1, r=0}^{N, Z_{S}^{[T]}} \mid \varphi_{i, r}^{[T]} \geq S_{n, r}^{[T]}, \sum_{r=0}^{Z_{S}^{[T]}} \varphi_{i, r}^{[T]}=1\right\} .
\end{gathered}
$$

\section{B. Mixed Strategy NE Solution}

In this subsection, we begin by finding the mixed-strategy NE solution to $\mathfrak{C B}_{2}$, which is defined as follows.

Definition $3(\mathbf{N E})$ : A strategy profile $\left(\mathbf{h}^{*}, \mathbf{s}^{*}\right)$ is a mixedstrategy NE point of $\mathfrak{C B}_{2}$ if and only if no player can improve its utility by deviating unilaterally, i.e.,

$$
\begin{gathered}
U_{H}^{[T]}\left(\mathbf{h}^{*}, \mathbf{s}^{*}\right) \geq U_{H}^{[T]}\left(\mathbf{h}, \mathbf{s}^{*}\right), \forall \mathbf{h} \in \mathbf{\Pi}_{H}, \\
U_{S}^{[T]}\left(\mathbf{h}^{*}, \mathbf{s}^{*}\right) \geq U_{S}^{[T]}\left(\mathbf{h}^{*}, \mathbf{s}\right), \forall \mathbf{s} \in \mathbf{\Pi}_{S} .
\end{gathered}
$$


For ease of exposition, we consider $\mathfrak{C B}_{2}$ with asymmetric players, in which the HAP and the SA have different numbers of tile grids, i.e., $Z_{H}^{[T]}>Z_{S}^{[T]}$, and both of which deploy tile grids on battlefield set $\mathcal{N}$ during time horizon $T$. We use $\mathbf{1}_{h \times s}$ and $\mathbf{0}_{h \times s}$ to denote the all-1 and all- $h \times s$ matrix, respectively. Let $\lfloor\cdot\rfloor$ be the lower floor function. Thereby, the mixed-strategy $\mathrm{NE}$ point of $\mathfrak{C B}_{2}$ is specified in the following.

Theorem 1: If $\frac{2}{N} \leq \frac{Z_{S}^{[T]}}{Z_{H}^{[T]}} \leq 1$ and $N \geq 3,\left(\mathbf{h}^{*}, \mathbf{s}^{*}\right)$ constitutes a mixed-strategy $\mathrm{NE}$ point of $\mathfrak{C B}_{2}$, where

$$
\begin{aligned}
& \mathbf{h}^{*}=\left[\begin{array}{lll}
\mathbf{0}_{N \times 1} & \frac{\mathbf{1}_{N \times\lfloor 2 \Xi\rfloor}}{\lfloor 2 \Xi\rfloor} & \mathbf{0}_{N \times\left(Z_{H}^{[T]}-\lfloor 2 \Xi\rfloor\right)}
\end{array}\right],
\end{aligned}
$$

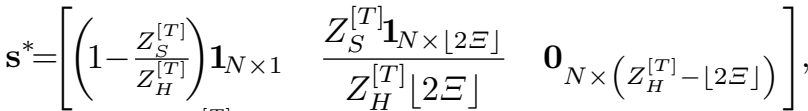

where $\Xi=\frac{Z_{H}^{[T]}}{N}$.

Proof: Due to the asymmetric players, $\mathfrak{C}_{2}$ can be formulated as a CBG with asymmetric resources over battlefield set $\mathcal{N}$ during $T$. The mixed-strategy $\mathrm{CBG}$ game has a NE $\left(\mathbf{h}^{*}, \mathbf{s}^{*}\right)$, where $\mathbf{h}^{*}$ (or $\mathbf{s}^{*}$ ) is the best distribution probability in the distribution vector $\mathbf{H}$ (or $\mathbf{S}$ ). As stated in [15], the HAP (or the SA) chooses the probability density function $\mathcal{Z}_{n}^{H}\left(H_{n}^{*}\right)$ (or $\mathcal{Z}_{n}^{S}\left(S_{n}^{*}\right)$ ) according to the allocated resource budget of tile grids, for $H_{n}^{*}, S_{n}^{*} \in\left[0,\left\lfloor\frac{2 Z_{H}^{[T]}}{N}\right\rfloor\right]$, which can be calculated as

$$
\begin{aligned}
& \mathcal{Z}_{n}^{H}\left(H_{n}^{*}\right)=\frac{H_{n}^{*}}{\left\lfloor\frac{2 Z_{H}^{[T]}}{N}\right\rfloor}, \\
& \mathcal{Z}_{n}^{S}\left(S_{n}^{*}\right)=\left(1-\frac{Z_{S}^{[T]}}{Z_{H}^{[T]}}\right) \mathscr{F}\left(S_{n}^{*}\right)+\left(\frac{Z_{S}^{[T]}}{Z_{H}^{[T]}}\right) \frac{S_{n}^{*}}{\left\lfloor\frac{2 Z_{H}^{[T]}}{N}\right\rfloor},
\end{aligned}
$$

where $\mathscr{F}(\cdot)$ corresponds to the unit pulse function. That is, if $S_{n}^{*}=0, \mathscr{F}\left(S_{n}^{*}\right)=1$; if $S_{n}^{*} \in\left[1,\left\lfloor\frac{2 Z_{H}^{[T]}}{N}\right\rfloor\right], \mathscr{F}\left(S_{n}^{*}\right)=0$.

By combining (15) and (21), the distributed vector $\mathbf{H}^{*}$ of the HAP to AUE $n$ is thus expressed by

$$
\mathbf{H}^{*}=\left\{H_{n}^{*}\right\} \sim \mathscr{U}\left(\left\{0,1,2, \cdots,\left\lfloor\frac{2 Z_{H}^{[T]}}{N}\right\rfloor\right\}\right),
$$

where $\mathscr{U}(\cdot)$ denotes the uniform distribution of the elements. Meanwhile, by jointly applying (16) and (22), we can obtain the distributed vector $\mathbf{S}^{*}$ of the SA to AUE $n$ as follows

$$
\begin{aligned}
\mathbf{S}^{*}=\left\{S_{n}^{*}\right\} \sim & \left(1-\frac{Z_{S}^{[T]}}{Z_{H}^{[T]}}\right) \mathscr{F}\left(S_{n}^{*}\right)+\left(\frac{Z_{S}^{[T]}}{Z_{H}^{[T]}}\right) \\
& \times \mathscr{U}\left(\left\{0,1,2, \cdots,\left\lfloor\frac{2 Z_{H}^{[T]}}{N}\right\rfloor\right\}\right) .
\end{aligned}
$$

As in [16], based on (23) and (24), the best distribution probability $\mathbf{h}^{*}$ of the HAP to AUE $n$ can be determined by

$$
\mathbf{h}^{*}=\left\{H_{n, r}^{*}\right\}=\frac{1}{\left\lfloor\frac{2 Z_{H}^{[T]}}{N}\right\rfloor}, 1 \leq r \leq\left\lfloor\frac{2 Z_{H}^{[T]}}{N}\right\rfloor .
$$

Similarly, we can also obtain the best distribution probability $\mathbf{s}^{*}$ of the SA to $\operatorname{AUE} n$, i.e.,

$$
\mathbf{s}^{*}=\left\{S_{n, r}^{*}\right\}= \begin{cases}1-\frac{Z_{S}^{[T]}}{Z_{H}^{[T]}}, & r=0, \\ \left(\frac{Z_{S}^{[T]}}{Z_{H}^{[T]}}\right) \frac{1}{\left\lfloor\frac{2 Z_{H}^{[T]}}{N}\right\rfloor_{Z^{[T]}}}, & 1 \leq r \leq\left\lfloor\frac{2 Z_{H}^{[T]}}{N}\right\rfloor .\end{cases}
$$

For notational simplicity, let $\Xi=\frac{Z_{H}^{[T]}}{N}$. This concludes the proof of our theorem.

Corollary 1 . With the $\mathrm{NE}\left(\mathbf{h}^{*}, \mathbf{s}^{*}\right)$ to $\mathfrak{C B}_{2}$, the expected privacy protection level is expressed by $1-Z_{S}^{[T]} / Z_{H}^{[T]}$, and the expected total utilities of two players are achieved as

$$
U_{H}^{[T]}\left(\mathbf{h}^{*}, \mathbf{s}^{*}\right)=-U_{S}^{[T]}\left(\mathbf{h}^{*}, \mathbf{s}^{*}\right)=\left(1-\frac{Z_{S}^{[T]}}{Z_{H}^{[T]}}\right) \sum_{n=1}^{N} \epsilon_{n}^{1} .
$$

Proof: At the mixed-strategy NE to $\mathfrak{C B}_{2}$, the number of best assigned tile grids for each AUE between the HAP and the SA have different cases with $H_{n}^{*}>S_{n}^{*}$ and $H_{n}^{*} \leq S_{n}^{*}$, and the best probability in different cases are denoted by $\operatorname{Pr}\left(H_{n}^{*}>S_{n}^{*}\right)$ and $\operatorname{Pr}\left(H_{n}^{*} \leq S_{n}^{*}\right)$, respectively. Referring to (11), only if $H_{n}^{*}>$ $S_{n}^{*}$, the HAP can obtain the best expected total utilities, i.e., $\operatorname{Pr}\left(H_{n}^{*}>S_{n}^{*}\right)$. Based on (10), (14), (19), and (20), we derive

$$
\begin{aligned}
& \mathbb{E}_{\left(\mathbf{h}^{*}, \mathbf{s}^{*}\right)}\left[\sum_{n=1}^{N}\left\{\epsilon_{n}^{1} F\left(H_{n}^{[T]}, S_{n}^{[T]}\right)+\epsilon_{n}^{2}\left[1-F\left(H_{n}^{[T]}, S_{n}^{[T]}\right)\right]\right\}\right] \\
= & \sum_{n=1}^{N} \epsilon_{n}^{1}\left(\operatorname{Pr}\left(H_{n}^{*}>S_{n}^{*}\right) \mid S_{n}^{*}=0, S_{n}^{*} \neq 0\right) \\
= & \sum_{n=1}^{N} \epsilon_{n}^{1}\left[\operatorname{Pr}\left(H_{n}^{*}>0\right)+\left(\operatorname{Pr}\left(H_{n}^{*}>S_{n}^{*}\right)\right) \mid S_{n}^{*} \neq 0\right] \\
= & \left(1-\frac{Z_{S}^{[T]}}{Z_{H}^{[T]}}\right) \sum_{n=1}^{N} \epsilon_{n}^{1} .
\end{aligned}
$$

As a result, Corollary $\mathbf{1}$ is derived.

Remark: In this work, our goal is to achieve the improvement of the total utilities by effectively identifying the tradeoff between privacy protection level and energy consumption in laser-powered aerial MEC system. When $Z_{H}^{[T]}>Z_{S}^{[T]}$, the HAP guarantees that more AUEs will obtain the laser-enabled WPT, which ensures that more computing tasks can be completed locally or offloading to the HAP. In other words, the HAP wins the game and the total utilities increase accordingly with the growing number of tile grids allocated by the HAP.

\section{Simulation Results}

In this section, we provide simulation results to validate the performance of our proposed game framework. We consider a laser-powered aerial MEC system consisting of a HAP deployed in the stratosphere at altitude $L_{\mathrm{H}}=21 \mathrm{~km}, N=15$ AUEs with randomly flight trajectories within the considered airspace $\Omega$, and a fixed SA on the ground. The time horizon $T=2 \mathrm{~s}$ is divided into $K=5$ slots. The considered airspace $\Omega$ is discretized into $Y=11$ tiers. All the tiers are assumed to be evenly spaced by vertical distance $L_{\mathrm{T}}=200 \mathrm{~m}$, and the vertical distance is set to $L_{0}=1 \mathrm{~km}$ between the lowest tier and the Earth's surface. In addition, each tier is composed of $120 \times 120$ square tile grids with the unit length of one side, i.e., $1 \mathrm{~m}$. All the AUEs adopt the Ka-band sharing the same 
TABLE I

SimUlation PARAMETERS.

\begin{tabular}{|l|l|l|}
\hline Parameter & Description & Value \\
\hline \hline$\beta$ & Fixed transmit power of laser & $5.7 \times 10^{13} \mathrm{~W}$ \\
\hline$\tau$ & Energy conversion efficiency & 0.7 \\
\hline$A$ & Area of mirror & $7.85 \times 10^{-3} \mathrm{~m}^{2}$ \\
\hline$D$ & Size of the initial laser beam & $0.1 \mathrm{~m}$ \\
\hline$\Delta \theta$ & Angular spread & $3.4 \times 10^{-5}$ \\
\hline$\chi$ & Combined optical efficiency & $1.5 \mathrm{Lm} / \mathrm{W}$ \\
\hline$\zeta$ & Attenuation coefficient & $10^{-6}$ \\
\hline$\phi_{n}$ & Carrier frequency & $3 \mathrm{GHz}$ \\
\hline$\alpha$ & Path loss exponent & 2 \\
\hline$l_{\mathrm{A}}$ & Atmospheric loss & $1.2 \mathrm{~dB}$ \\
\hline$\sigma^{2}$ & Variance of the AWGN & $-30 \mathrm{dBm}$ \\
\hline$f_{n}$ & Local computing capability & $2 \times 10^{9} \mathrm{cycles} / \mathrm{s}$ \\
\hline$\kappa$ & Effective switched capacitance & $10^{-26}$ \\
\hline$\ell_{1}$ & Parameter related to AUE & $9.26 \times 10^{-3} \mathrm{~kg} / \mathrm{m}$ \\
\hline$\ell_{2}$ & Parameter related to AUE & $3500 \mathrm{~kg} \cdot \mathrm{m}^{3} / \mathrm{s}^{4}$ \\
\hline$m$ & Mass of all the payloads & $10 \mathrm{~kg}$ \\
\hline$g$ & Gravity acceleration & $9.8 \mathrm{~m} / \mathrm{s}^{2}$ \\
\hline
\end{tabular}

$\mathrm{RB}$ to communicate with the HAP via A2A LOS links. For simplicity, the transmit power of each AUE is assumed to be randomly assigned by the range of $[250 \mathrm{~mW}, 400 \mathrm{~mW}]$ at each slot. Each orthogonal subchannel owns the equal bandwidth of $W=40 \mathrm{MHz}$. For computing model, we set the A2A uplink transmission overhead to $\eta_{n}=150 \mathrm{bits}$ for each AUE. The number of total input-bits for each AUE $B_{n}[k]$ and input-bits for offloading from each AUE to the HAP $\xi_{n}[k]$ are assigned to $[2.8 \mathrm{~GB}, 3.2 \mathrm{~GB}]$ and $[2 \mathrm{~GB}, 3 \mathrm{~GB}]$ at each slot, respectively. Furthermore, we assume that each AUE is flying at a constant speed at each slot. For each AUE, the velocity vector $\mathbf{v}[k]$ is selected randomly from $[(8,8),(15,20)]$ and the acceleration vector $\mathbf{a}[k]$ are always set to $(0,0)$. The positive weighting factor are set to $\lambda_{1}=0.9$, and $\lambda_{2}=10^{-15}$, respectively. Other relevant parameters in simulations are listed in Table I.

In Fig. 2(a), we examine the impact of the vertical distance between AUE $n$ and the HAP on the privacy protection level of AUE $n$. It can be seen that the privacy protection level decreases with the growth of vertical distance. The reason is that the more CPU cycles it takes to calculate one bit, the less bits are calculated during $T$. Besides, the privacy protection level clearly decreases with the increase of the number of CPU cycles required to process one bit of input data $\varpi_{n}$. Fig. 2(b) shows the performance comparison of the total utilities that the HAP receives from all the AUEs versus the number of tile grids. We can observe that the total utilities obtained by the HAP increases consistently with the growing number of tile grids. Moreover, when the number of CPU cycles required to process one bit of input data $\varpi_{n}$ decreases, the total utilities obtained by the HAP will increase. This highlights the importance of properly tuning the number of CPU cycles allowing, theoretically, the better total utilities for the HAP.

\section{CONClusion}

This paper investigated the new privacy issue of charging location for the AUEs during the WPT signaling delivery in the laser-powered aerial MEC system. We formulated the interaction between the HAP and the SA in their allocation of tile grids to the AUEs as the CBG, which characterizes the competition of two players for limited resources over a set
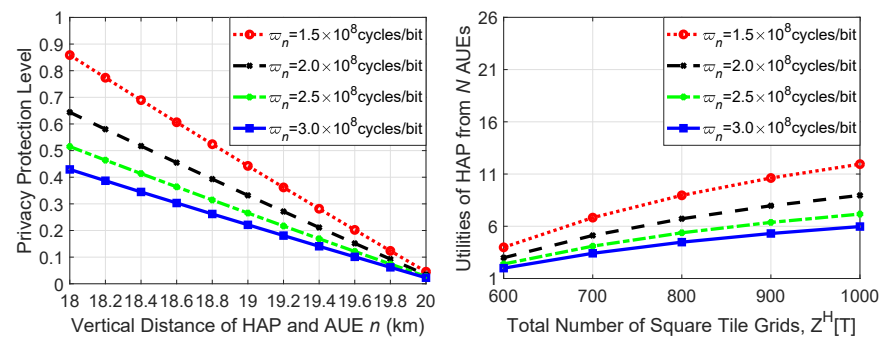

(a) Privacy protection level of AUE $n$. (b) Utilities of HAP from $N$ AUEs.

Fig. 2. Performance of the proposed game framework.

of battlefields. By considering with asymmetric players, the mixed-strategy NE solution was derived. Simulation results demonstrated the effectiveness of our proposed game framework, which ensures an enhanced privacy for laser-enabled WPT in aerial MEC system. Future work will continue to weigh the delay to improve the utility of the entire system.

\section{REFERENCES}

[1] L. Zhang, H. Zhao, S. Hou, Z. Zhao, H. Xu, X. Wu, Q. Wu, and R. Zhang, "A survey on 5G millimeter wave communications for UAVassisted wireless networks," IEEE Access, vol. 7, pp. 117460-117 504, Jul. 2019.

[2] M. Mozaffari, A. T. Z. Kasgari, W. Saad, M. Bennis, and M. Debbah, "Beyond 5G with UAVs: Foundations of a 3D wireless cellular network," IEEE Trans. Wireless Commun., vol. 18, no. 1, pp. 357-372, Jan. 2019.

[3] L. Zhang, H. Zhang, C. Guo, H. Xu, L. Song, and Z. Han, "Satelliteaerial integrated computing in disasters: User association and offloading decision," in Proc. IEEE ICC, Dublin, Ireland, Jun. 2020.

[4] J. Moura and D. Hutchison, "Game theory for multi-access edge computing: Survey, use cases, and future trends," IEEE Commun. Surveys Tuts., vol. 21, no. 1, pp. 260-288, Firstquarter 2019.

[5] W. Jaafar and H. Yanikomeroglu, "Dynamics of quadrotor UAVs for aerial networks: An energy perspective," arXiv preprint arXiv:1905.06703, May 2019.

[6] J. Feng, Q. Pei, F. R. Yu, X. Chu, and B. Shang, "Computation offloading and resource allocation for wireless powered mobile edge computing with latency constraint," IEEE Wireless Commun. Lett., vol. 8, no. 5, pp. 1320-1323, Oct. 2019.

[7] S. Mao, S. Leng, S. Maharjan, and Y. Zhang, "Energy efficiency and delay tradeoff for wireless powered mobile-edge computing systems with multi-access schemes," IEEE Trans. Wireless Commun., vol. 19, no. 3, pp. 1855-1867, Mar. 2020.

[8] Y. Liu, K. Xiong, Q. Ni, P. Fan, and K. B. Letaief, "UAV-assisted wireless powered cooperative mobile edge computing: Joint offloading, CPU control, and trajectory optimization," IEEE Internet Things J., vol. 7, no. 4, pp. 2777-2790, Apr. 2020.

[9] J. Ouyang, Y. Che, J. Xu, and K. Wu, "Throughput maximization for laser-powered UAV wireless communication systems," in Proc. IEEE ICC Workshops, Kansas City, MO, USA, May 2018.

[10] M. Mori, H. Kagawa, and Y. Saito, "Summary of studies on space solar power systems of Japan Aerospace Exploration Agency (JAXA)," Acta Astronautica, vol. 59, no. 1-5, pp. 132-138, Jul. 2006.

[11] M.-A. Lahmeri, M. A. Kishk, and M.-S. Alouini, "Stochastic geometrybased analysis of airborne base stations with laser-powered UAVs," IEEE Commun. Lett., vol. 24, no. 1, pp. 173-177, Jan. 2020.

[12] Q. Zhang, W. Fang, Q. Liu, J. Wu, P. Xia, and L. Yang, "Distributed laser charging: A wireless power transfer approach," IEEE Internet Things J., vol. 5 , no. 5, pp. 3853-3864, Oct. 2018.

[13] L. Xiao, C. Xie, M. Min, and W. Zhuang, "User-centric view of unmanned aerial vehicle transmission against smart attacks," IEEE Trans. Veh. Technol., vol. 67, no. 4, pp. 3420-3430, Apr. 2018.

[14] Y. Zeng and R. Zhang, "Energy-efficient UAV communication with trajectory optimization," IEEE Trans. Wireless Commun., vol. 16, no. 6, pp. 3747-3760, Jun. 2017.

[15] B. Roberson, "The Colonel Blotto game," Economic Theory, vol. 29, no. 1, pp. 1-24, Jan. 2006.

[16] S. Hart, "Discrete Colonel Blotto and General Lotto games," Int. J. Game Theory, vol. 36, no. 3-4, pp. 441-460, Oct. 2007. 